\title{
The role of the chemokine receptor XCRI in breast cancer cells
}

This article was published in the following Dove Press journal:

Breast Cancer - Targets and Therapy

29 March 2017

Number of times this article has been viewed

\section{Xiao Li Yangl,* \\ Li Guo Qi ${ }^{2}$,* \\ Feng Juan Lin' \\ Zhou Luo Ou'}

'Department of Oncology, Breast Cancer Institute, Fudan University, Shanghai Cancer Center, Shanghai, ${ }^{2}$ Department of Neurosurgery, Taian City Central Hospital, Taian, Shangdong, People's Republic of China

*These authors contributed equally to this work
Correspondence: Zhou Luo Ou Department of Oncology, Breast Cancer Institute, Fudan University Shanghai Cancer Center, 270 DongAn Road, Shanghai 200032, People's Republic of China

Tel +862164175590

Fax +862164174774

Email ouzhouluo@163.com
Abstract: Considerable attention has recently been paid to the application of chemokines to cancer immunotherapy due to their complex role in cell proliferation, invasion, metastasis, and tumorigenesis, which extends beyond the regulation of lymphocyte migration during immune responses. The expression and the function of the chemokine receptor XCR1 on breast cancer have remained elusive to date. In this study, the expressions of XCR1 mRNA were tested by quantitative real-time polymerase chain reaction in one breast epithelial cell line (MCF-10A) and nine breast cancer cell lines (MDA-MB-231, 231HM, 231BO, MDA-MB-468, MCF-7, T47D, Bcap-37, ZR-75-30, and SK-BR-3). We established XCR1-overexpressing breast cancer cell line MDA-MB-231 (231/XCR1) in XCR1 low expression cell line MDA-MB-231 (231). The ability of proliferation, invasion, and metastasis was measured by CCK8, plate cloning formation, and transwell analysis, respectively, in XCR1-overexpressing breast cancer cell lines (231/XCR1) and their parental cell line MDA-MB-231/Vector (simplified as "231/Vector"); $5 \times 10^{6} / 100 \mu \mathrm{L}$ cells were inoculated in mammary fat pad of BALB/c nude mice. There were six BALB/c nude mice in the experimental group and control group. Protein expression was analyzed by cell immunofluorescence and Western blot. The growth of XCR1-overexpressing human breast cancer cell line MDA-MB-231 in vitro was restrained and tumorigenesis in vivo was also extenuated, its mechanism may involve in the inhibition of MAPK and PI3K/AKT/mTOR signaling pathway, but increase in LC3 expression. However, the overexpression of XCR1 in human breast cancer cell line MDA-MB-231 in vitro can promote the migration and invasion partially due to decreasing the protein level of $\beta$-catenin. Therefore, XCR1 can affect the biological characteristics of some special breast cancer cells through complex signal transduction pathway.

Keywords: breast cancer, function, XCR1, proliferation

\section{Introduction}

Despite progress in the detection and treatment of breast cancer, this disease is still one of the most common malignancy among women worldwide and is the second cause of incidence rates among all the cancers in China, with both increasing incidence and mortality. ${ }^{1}$ More effective molecular targets of carcinogenesis and metastasis in breast cancer are still needed.

The chemokine ligand-receptor interactions are important in immune surveillance, inflammation, angiogenesis, as well as tumor growth and metastasis. ${ }^{2,3}$ Although most chemokines are members of the CC or CXC subgroups, XCL1, also known as lymphotactin, is the sole member of the $\mathrm{C}$ subgroup. ${ }^{4} \mathrm{X}-\mathrm{C}$ motif chemokine receptor 1 (XCR1), as the receptor of XCL1, is the only chemokine receptor selectively expressed in "Batf3-IRF-8-Id2-dependent dendritic cells (DCs)" or as "CD8 alpha (+) 
conventional DCs" that is potent in antigen presentation to $\mathrm{T}$ cells and facilitates cytotoxic T-cell response. XCR1, thus, emerges as the first surface marker characterizing a DC lineage in the mouse and potentially also in the human. ${ }^{5}$ XCL1 is produced by activated CD8 (+) T cells, natural killer (NK) cells, gamma delta T cells, and mast cells. NK and CD8 (+) $\mathrm{T}$ cells increased XCL1 production upon activation. Thus, certain DC subsets should be chemotactic toward NK or activated CD8 (+) T cells through XCR1. ${ }^{2,6}$ Therefore, it is considered significant in inflammation and allergic disease, such as in human allergic asthma, ${ }^{7}$ experimental autoimmune anterior uveitis, ${ }^{8}$ osteoarthritis and rheumatoid arthritis, ${ }^{9}$ and the chronic stage of infection with Mycobacterium tuberculosis, ${ }^{10,11}$ because of the recruitment of regulatory cell subsets and enhancement of their activities, which are mediated by the interaction between XCL1 and XCR1.

Similar to other family members, XCR1 also have implicated functions in different kinds of human tumors. For example, Khurram et $\mathrm{l}^{12}$ reported that XCR1 mRNA and cell surface protein expression in oral squamous cell carcinoma cell lines showed a greater response to lymphotactin than normal keratinocytes, indicating that it may play an important role in cell migration, invasion, and proliferation. Kim et $\mathrm{al}^{13}$ found that XCR 1 was expressed in primary and metastatic human epithelial ovarian carcinoma (EOC) specimens and cell lines, but not detected in the normal ovary or in human normal ovarian surface epithelial cells, suggesting that the expression of XCR1 was related to cell carcinogenesis and migration. In another study, the expression of the chemokine receptor XCR1 and frequent usage of autoreactive $\mathrm{V}(\mathrm{H})$ genes seem to be distinct characteristics of diffuse large B-cell lymphoma initially manifesting in the bone marrow. ${ }^{14}$ Thus, XCR1 seems to induce cancer cell growth, migration, and invasion. Although XCL1 was found to be expressed in mammary gland, the expression and function of XCR1 in breast cancer are still unknown.

In this study, we used a stable expressing XCR1 breast cancer cell line 231/XCR1 derived from parent MDAMB-231 cells to investigate the possible role of XCR1 in the proliferation, migration, and invasion in vitro and in vivo. Interestingly, we found XCR 1 is closely related to the expression of estrogen receptor (ER). In addition, XCR1 can suppress breast cancer growth, tumorigenesis at least in part, through MAPK and PI3K/AKT/mTOR signaling pathways, but promote metastasis via decreasing the protein level of $\beta$-catenin.

\section{Materials and methods}

\section{Cell line and culture}

Human breast cancer cell lines, MDA-MB-231, MDAMB-468, ZR-75-30, T474D, MCF-7, ZR-75-30, and SK-BR-3, and one normal breast epithelial cell line, namely MCF-10A, were obtained from the American Type Culture Collection (Manassas, VA, USA).

MDA-MB-231HM and MDA-MB-231BO cell lines were established by subclone selection procedure in our institute. The MDA-MB-231HM cell line has a high potential to metastasize to the lung, its establishment has been described previously ${ }^{15}$ and MDA-MB-231BO is more likely to metastasize to the bone. Cells were routinely maintained in the recommended medium supplemented with $10 \%$ fetal bovine serum, $100 \mathrm{U} / \mathrm{mL}$ of penicillin, and $100 \mu \mathrm{g} / \mathrm{mL}$ of streptomycin. The cultures were incubated at $37^{\circ} \mathrm{C}$ in a humidified $5 \% \mathrm{CO}_{2}$ atmosphere. Cell culture medium and fetal bovine serum were purchased from Thermo and Invitrogen (Carlsbad, CA, USA). Real-time polymerase chain reaction (RT$\mathrm{PCR}$ ) reagents were from TaKaRa Bio Inc. (Kusatsu, Japan).

\section{RNA extraction, real-time quantitative PCR}

TRIzol reagent (Invitrogen) was used to extract total RNA according to the manufacture's recommendations. In brief, $1.0 \mu \mathrm{g}$ of total RNA was applied for reverse transcription in $20 \mu \mathrm{L}$. The specific primers used in the PCR were as follows: XCR1: forward 50-CGCGGATCCATGGAGTCCTCAGGCAACCCAGAGA-30, reverse 50-ACGCGTCGACTCAGTAGAAGGAGGCGCCCTCA; glyceraldehyde 3-phosphate dehydrogenase (GAPDH): forward 50-AAGGTGAAGGTCGGAGTCAAC-30, reverse 50-GGGGTCATTGATGGCAACAATA. The process of PCR was performed according to the protocol of the kit from TaKaRa Bio Inc.

The quantification of mRNA levels was carried out using DNA Engine Opticon 2 real-time PCR detection system (MJ Research Inc., Waltham, MA, USA) with SYBR Green. Two microliters of diluted cDNA were used as a template; $10 \mu \mathrm{L} \times 2$ SYBR Premix Ex Taq (TaKaRa Bio Inc.) were mixed as template and primers. The total reaction volume was $20 \mu \mathrm{L}$. Cycling conditions were denaturation at $95^{\circ} \mathrm{C}$ for $30 \mathrm{~s}$, annealing at $60^{\circ} \mathrm{C}$ for $30 \mathrm{~s}$, and elongation at $72^{\circ} \mathrm{C}$ for $45 \mathrm{~s}$. Plate reading was at $72^{\circ} \mathrm{C}$ and $82^{\circ} \mathrm{C}$. The specific primers used in the experiment were as follows: $\mathrm{XCR} 1$ : forward 5'-CTGTTCTGCTACGTGGAGATCC-3', reverse 5'-CAGGGCGTATTCTAGCTGCTG-3'; GAPDH: forward 
5'-ACCCACTCCTCCACCTTTGA-3', reverse 5'-CATACCAGGAAATGAGCTTG ACAA-3'. All experiments were repeated in triplicate. To ensure that the correct product was amplified in the reaction, all PCR products were also separated on $1.2 \%$ agarose gel electrophoresis.

\section{Cloning of XCRI cDNA and viral infection}

Trizol reagent (Invitrogen) was used to extract total RNA according to the manufacture's recommendations. XCR1 cDNA was produced by reverse transcription-PCR. First, reverse transcription was performed with random hexamer primers by using the Ominoscript RT kit (TaKaRa Bio Inc.). The primers used to amplify XCR1 cDNA were 5'-CGCGG ATCCATGGAGTCCTCAGGCAACCCAGAGA-3'(sense, boldface type indicates the BamHI site) and 5'-ACGCGTCGACTCAGTAGAAGGAGGCGCCCTCA-3' (antisense, boldface type indicates the SalI site). Each cycle consisted of denaturing at $94^{\circ} \mathrm{C}$ (for $30 \mathrm{~s}$ ), annealing at $56^{\circ} \mathrm{C}$ (for $1 \mathrm{~min}$ ), and extension at $72^{\circ} \mathrm{C}$ (for $1 \mathrm{~min}$ ) for a total of 35 cycles. The XCR1 reverse transcription-PCR product was purified in a $1.2 \%$ agarose gel, digested with BamHI and SalI, and ligated to a $\mathrm{pBabe/puromycin} \mathrm{retroviral} \mathrm{vector} \mathrm{that} \mathrm{had} \mathrm{been}$ digested with the same enzymes. After transformation of the plasmid in Escherichia coli (DH5 $\alpha$ ), positive clones were selected and DNA sequencing analysis was performed at the DNA sequencing corporation. The control vector used in this study was an empty $\mathrm{pBabe/puromycin} \mathrm{retroviral} \mathrm{vector.} \mathrm{All}$ these plasmids were transfected into amphotropic Phoenix packaging cells to generate retroviruses, which were used to infect corresponding cells lines. Retroviruses carrying XCR1 cDNA were used to infect MDA-MB-231 cells.

\section{Western blot}

Western blot analysis was performed according to the standard protocols. Briefly, aliquots of total protein $(30 \mu \mathrm{g})$ were electrophoresed on sodium dodecyl sulfate polyacrylamide, appropriate Tris- $\mathrm{HCl}$ gels. The separated proteins were transferred to polyvinylidene difluoride membranes (Millipore Co, Billerica, MA, USA) and incubated with primary antibodies for $2 \mathrm{~h}$. Chemiluminescent detection was performed, and images were captured by LAS-3000 system (Fujifilm, Tokyo, Japan). Antibodies against $\beta$-catenin (51067-2-AP1:800), LC3A/B (66139-1-1g, 1:1000), and GAPDH (60004-1Ig, 1:1000) were from Proteintech. Antibodies against MEK1/2 (9126, 1:1000), Phospho-MEK1/2 (2338, 1:1000), PhosphoERK1/2 (4376, 1:1000), AKT (4691.1:1000), Phospho-AKT
(4060P,1:1000), Phospho-P38 (4511.1:1000), Phospho-JNK (4668,1:1000), E-cadherin (3195,1:1000), Phospho-p53 (9284.1:1000), and mTOR Substrates Sampler kit (CST 9862) that conclude Phospho-mTOR, mTOR, Phospho-p70s6389 (Thr389), Phospho-P70389 (Thr371), Phospho-4EBP1 (Thr37/46), and anti-rabbit IgG were from Cell Signaling Technology. Antibodies against p38 (sc-7149,1:1000), P53 (sc-126,1:1000), and JNK (sc-571, 1:1000) were from Santa Cruz Biotechnology (Dallas TX, USA).

\section{Proliferation assay}

Cell proliferation was detected by using Cell Counting Kit- 8 (Dojindo, Molecular Technologies Inc, Gaithersburg, MD, USA). Cells were plated in 96-well plates at a density of 2500 per well $(100 \mu \mathrm{L})$ and cultured in growth medium. The number of the cells was counted according to the protocol of the kit from the company.

\section{Cell colony formation}

A total of 250 cells were cultured in the recommended medium (DMEM) supplemented with 10\% fetal bovine serum, $100 \mathrm{U} / \mathrm{mL}$ penicillin, and $100 \mu \mathrm{g} / \mathrm{mL}$ streptomycin, which was maintained for 14 days at $37^{\circ} \mathrm{C}$ in an atmosphere of $5 \% \mathrm{CO}_{2}$ and $95 \%$ air. Then fixed/permeabilized the cells with $4 \%$ paraformaldehyde for $30 \mathrm{~min}$, fixative was removed, and cells were washed in phosphate-buffered saline (PBS) for three times and dyed with crystal violet for 30 min using PBS washed cells. The number of colonies that was larger than $50 \mu \mathrm{m}(\sim 100$ cells $)$ in diameter in each dish was counted. The assay was repeated three times with duplicate samples.

\section{Immunocytochemistry for XCR I}

Cells were grown on chamber slides (BD, Franklin Lakes, NJ, USA) and fixed/permeabilized with $4 \%$ paraformaldehyde containing $0.5 \%$ Triton-X100 (Sigma, Dorset, UK) for $20 \mathrm{~min}$. Fixative was removed, slides were washed in PBS, and rabbit anti-human XCR1 antibody ( $20 \mu \mathrm{g} / \mathrm{mL}$, LS-C178854, LifeSpan BioSciences) was added for $1 \mathrm{~h}$. Excess antibody was removed, and fluorescein isothiocyanate-conjugated secondary antibody was applied (1:1000 dilution, A-21430, Invitrogen) for $30 \mathrm{~min}$. Slides were washed again and mounted using Vectashield mounting medium containing 4',6-diamidine-2'-phenylindole dihydrochloride (DAPI; Vector Labs., Burlingame, CA, USA).

\section{Migration assay}

Chemotaxis assays were performed using transwell insert polycarbonate membranes ( $8 \mu \mathrm{m}$ pore size, $\mathrm{BD}) ; 5 \times 10^{4}$ cells 
were seeded in the top compartment, and $600 \mu \mathrm{L}$ DMEM with $10 \%$ fetal bovine serum was added to the bottom at $37^{\circ} \mathrm{C}$ for $13 \mathrm{~h}$. Nonmigratory cells on the upper membrane surface were removed, and migratory cells attached to the bottom surface of the membrane were fixed with $10 \%$ formalin and stained with $0.1 \%$ crystal violet for $30 \mathrm{~min}$ at room temperature. Migrated cells in five random fields were counted using a light microscope at a magnification of $\times 200$. All assays were performed in triplicate.

\section{Invasion assay}

Invasion experiments were carried out with a Matrigel invasion chamber (BD Labware). Each well insert was layered with $75 \mu \mathrm{L}$ of 1:4 mixture of Matrigel:DMEM. Serum-starved cells $\left(1 \times 10^{5}\right)$ were added to the upper compartment, and $600 \mu \mathrm{L}$ of DMEM with $10 \%$ fetal bovine serum was added to the bottom at $37^{\circ} \mathrm{C}$ for $24 \mathrm{~h}$. Invasion was assessed by counting the cells that had traveled across the filter and was attached to the bottom side of the filter. Then, the filter was fixed in $10 \%$ formalin and stained with $1 \%$ crystal violet. Cells that had invaded through the Matrigel and reached the lower surface of the filter were counted under a light microscope at a magnification of $\times 200$. Five fields were counted for each sample.

\section{Xenograft tumors in nude mice}

All mouse experiments were carried out in accordance with the National Institutes of Health (NIH) "Guide for the Care and Use of Laboratory Animals". The study protocol was approved by the Shanghai Medical Experimental Animal Care Committee. Breast tumor cells were implanted into the mammary fat pad as described previously. ${ }^{16}$ Briefly, we harvested $5 \times 10^{7}$ cells by incubation in trypsin-ethylenediaminetetraacetic acid, washed the cells twice with PBS, resuspended the cells in $1 \mathrm{~mL}$ of PBS. Cells $(0.1 \mathrm{~mL})$ were inoculated into the anesthetized mice. The tumor growth of modified and control cell lines was monitored until the day the mice were killed. The date on which the first grossly visible tumor appeared for subcutaneous injection was recorded, and the tumor size was measured every 3 days. Two-dimensional measurements were taken with an electronic caliper after injection, and tumor volume was calculated with the use of the following formula: tumor volume (in $\left.\mathrm{mm}^{3}\right)=a \times b^{2} \times 0.52$, where $a$ is the longest diameter; $b$, the shortest diameter; and 0.52 , a constant to calculate the volume of an ellipsoid. Mice were killed until observed for lethargy, poor appetite, and feebleness. Metastasis formation was assessed by macroscopic observation of all major organs for secondary tumors and confirmed by histological examination of organs. All tumor nodules were counted and dissected; each primary tumor nodule was also weighed and its volume was determined as described earlier for subcutaneous tumors.

\section{Immunohistochemical staining for animal xenografts}

Sections from xenografts of 231/XCR1 and 231/vector groups were used for LC3 and phospho-p53 protein detection. Antibodies used for immunohistochemical staining included anti-LC3 (66139-1-1g, 1:50, Proteintech), anti-phospho-p53 (9284.1:50, Cell Signaling Technology). Briefly, tissue slides were deparaffinized in xylene and rehydrated in a graded series of ethanol, and sections were subjected to antigen retrieval by boiling in $0.01 \mathrm{~mol} / \mathrm{L}$ sodium citrate buffer $(\mathrm{pH} 6.0)$ in a microwave oven for $10 \mathrm{~min}$. After blocking endogenous peroxidase activity with $0.3 \%$ hydrogen peroxide and blocking nonspecific protein binding with $1.5 \%$ normal goat serum, the sections were incubated overnight with an antibody at $4^{\circ} \mathrm{C}$ in a humid chamber. Then, antibodies were localized by incubating sections with biotinylated goat antimouse or goat antirabbit IgG for $30 \mathrm{~min}$ and detected with 3,3'-diaminobenzidine. The lung tissues were serially cut into $5 \mu \mathrm{m}$ slices, and every tenth section was stained with hematoxylin and eosin $(\mathrm{H} \& \mathrm{E})$ to evaluate the presence or absence of lung metastasis. Two independent pathologists calculated the number of metastasis in whole lungs.

\section{Statistical analysis}

Statistical analysis was performed using Statistical Package for the Social Sciences (SPSS) software Version 16.0 for Windows (SPSS Inc., Chicago, IL, USA). Analysis of variance (ANOVA) and Student's $t$-test were used to determine the statistical significance of differences between experimental groups in vitro. $p$-values of $<0.05$ were regarded as statistically significant. Graphs were created with GraphPad Prism 5.

\section{Ethical approval}

All applicable international, national, and institutional guidelines for the care and use of animal were followed. All animal experiments were approved by Shanghai Medical Experimental Animal Care Committee.

\section{Results}

\section{Expression of XCRI in human breast cancer cell lines}

The expressions of XCR1 mRNA were tested by quantitative RT-PCR in one breast epithelial cell line (MCF-10A) and nine breast cancer cell lines (MDA-MB-231, 231HM, 231BO, MDA-MB-468, MCF-7, T47D, Bcap-37, ZR-75-30, and SK-BR-3). We found that XCR1 mRNA expression is higher 
in ER+ breast cancer cell lines (MCF-7, T47D, Bcap-37, and ZR-75-30) than ER- breast cancer cell lines (MDA-MB-231 and MDA-MB-468; Figure 1A), which indicated a potential correlation between XCR1 expression and ER expression. To further confirm the association between XCR1 and ER, $\mathrm{XCR} 1$ protein expressions were detected by immunohistochemistry in 10 formalin-fixed, paraffin-embedded breast cancer tissue sections (five tissue sections are ER+ and the others are ER-). None of the patients had received preoperative chemotherapy or radiotherapy, and the levels of ER were approved in advance by the Institutional Review Board of the Fudan University Shanghai Cancer Center. Just as we expected, the expression of XCR1 protein is associated with ER expression (Figure 1B and C).

\section{Stable transfection of XCRI cDNA into MDA-MB-23I cells}

We suspected XCR1 may play a positive role in cell tumorigenesis and potential metastasis, so we transfected XCR1 expression vector $\mathrm{pBabe/XCR} 1$ into MDA-MB-231 cells and generated stable transfectants. We named the stable transfectants with high expression levels of XCR1 protein as $231 / \mathrm{XCR} 1$ and with empty vector as 231/Vector. As shown by reverse transcription-PCR (Figure 2A), real-time quantitative PCR (Figure 2B) and cell immunofluorescence, which was used to test the protein level of XCR1 (Figure 2C)

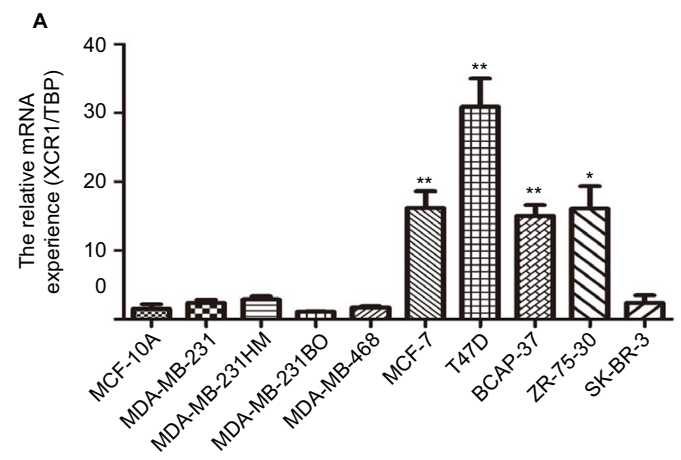

B
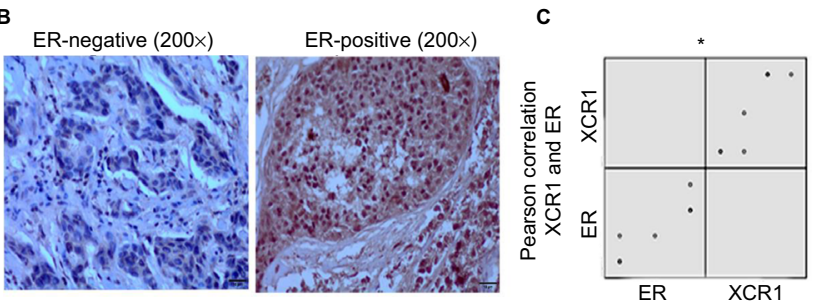

Figure I Expression of XCRI in breast cancer cell lines.

Notes: Expression of XCRI mRNA was measured by quantitative RT-PCR (A) in I normal breast epithelial cells and 9 breast cancer cell lines. XCRI protein expressions were detected by immunohistochemistry in 10 formalin-fixed, paraffin-embedded breast cancer tissue sections ( 5 tissue sections are ER+, the others are ER-), the expression of $X C R I$ protein is associated with ER expression (B, C). $* p<0.05, * * p<0.01$.

Abbreviations: ER, estrogen receptor; RT-PCR, real-time polymerase chain reaction.

as we showed, XCR1 was significantly overexpressed in 231/ XCR1 compared with 231/Vector.

\section{XCRI inhibits cell proliferation, tumorigenesis, and tumor growth}

To investigate whether XCR1 overexpression is associated with tumor growth in vitro, we performed the proliferation and anchorage-independent growth assay in XCR1transfected and their parental cells. As shown in Figure 3A, overexpression of XCR1 significantly slowed breast cancer proliferation and the smaller number of colonies formed in 231/XCR1 compared with control cells (Figure 3B and 3C). Next, the ability of XCR1 on tumor growth was further identified using an orthotropic xenograft tumor model in BALB/c mice. The results showed that when the mice were xenografted with 231/XCR1 cells, the primary tumor grew much smaller and slower than 231/vector cells in nude mice (Figure $3 \mathrm{D}$ and $3 \mathrm{E})$. Furthermore, the average rate of tumorigenesis in $231 / \mathrm{XCR} 1$ was only $50 \%$, whereas xenografts control xenografts was $100 \%$ (Figure 3F). The data, summarized in these figures, suggest that XCR1 inhibits ER-negative breast cancer cell proliferation, tumorigenesis, and tumor growth.

It has been shown that breast tumor cell proliferation is closely related to the activation of MAPK and PI3K/AKT/ mTOR pathways; therefore, we analyzed the relative protein expression. There was no change in total MEK, JNK, P38, and

A
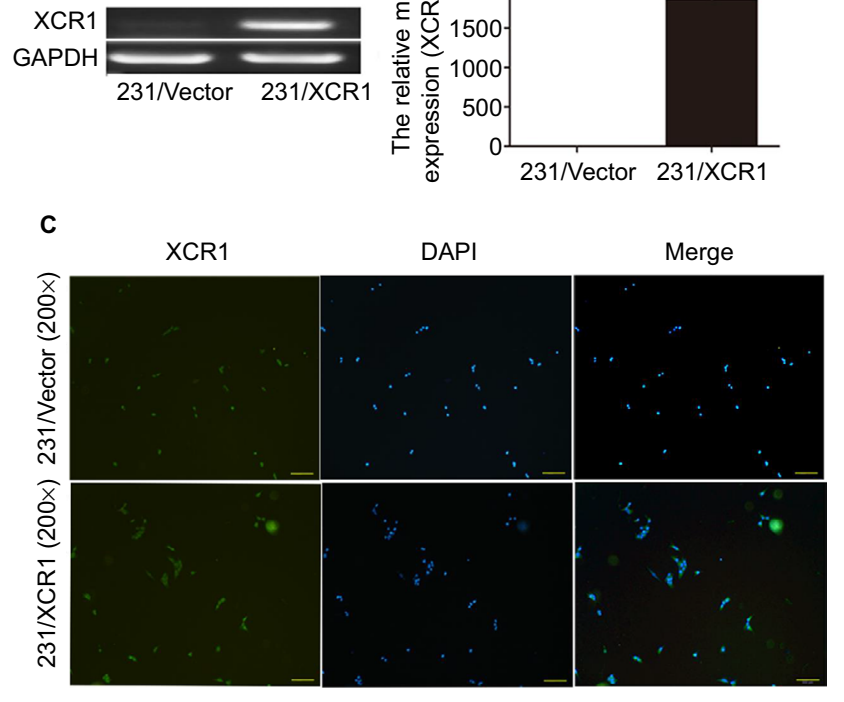

Figure 2 Stable transfection of XCRI cDNA (23I/XCRI) into MDA-MB-23 I cells. Notes: Expression of XCRImRNA was detected by RT-PCR (A) and quantitative RT-PCR (B) in MDA-MB-23I cells that were transfected with control (23I/vector) or XCRI cDNA $(23 I / X C R I)$. Expression of XCRI protein was detected by immunofluorescence in $23 \mathrm{I}$ /vector or $23 \mathrm{I} / \mathrm{XCRI}$ (C). ${ }^{* * *} p<0.00 \mathrm{I}$.

Abbreviations: RT-PCR, real-time polymerase chain reaction; DAPI, 4',6-diamidine2'-phenylindole dihydrochloride; GAPDH, glyceraldehyde 3-phosphate dehydrogenase. 
A
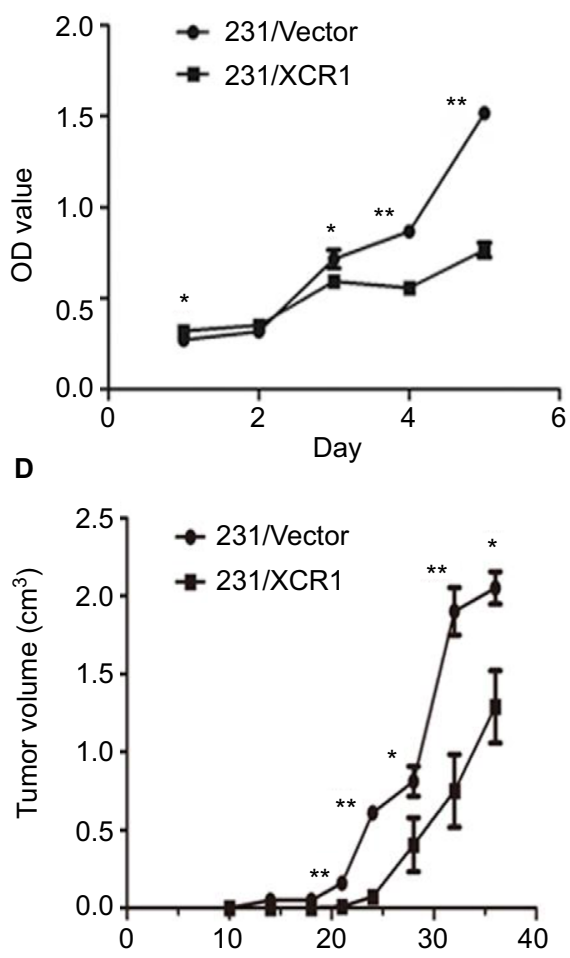

B
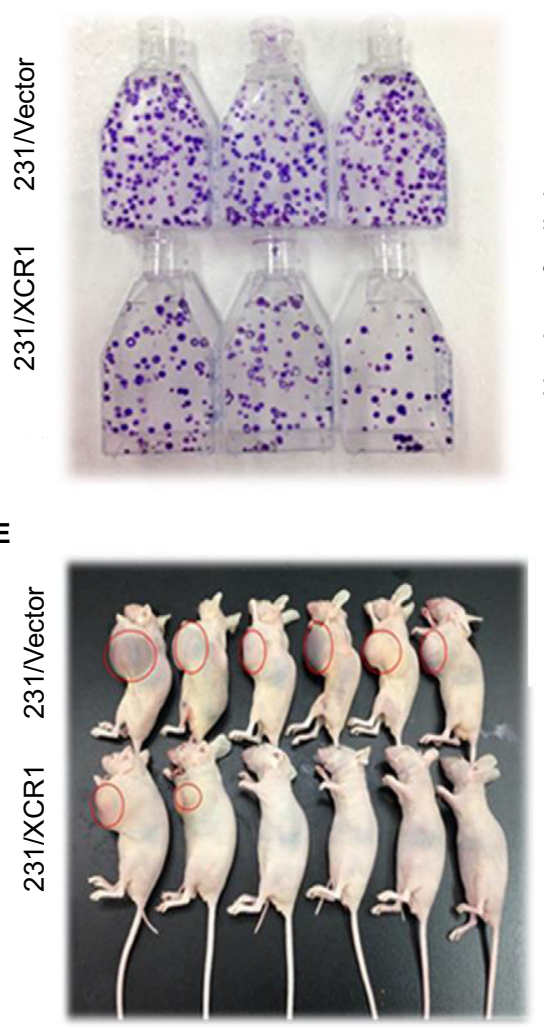

C

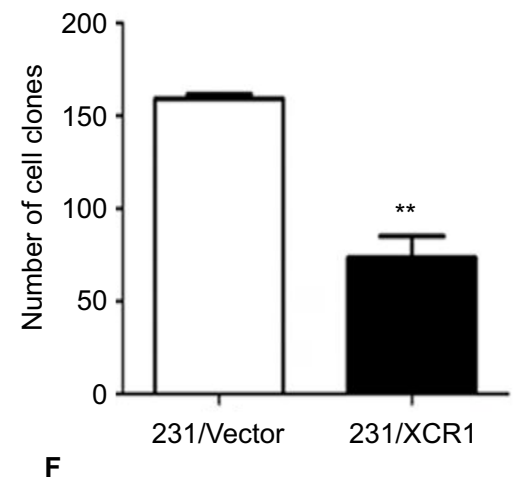

$\mathbf{F}$

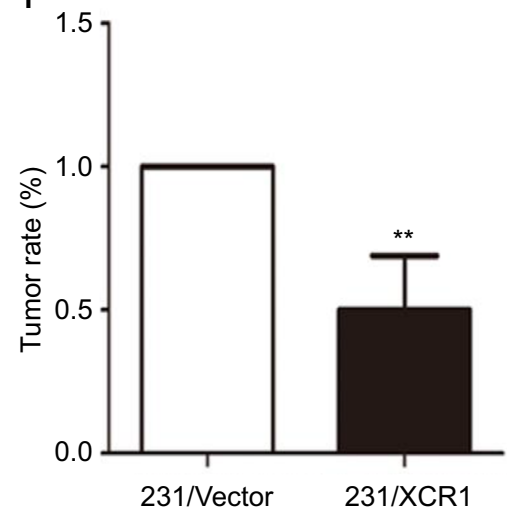

Figure 3 XCRI inhibits cell proliferation, tumorigenesis and tumor growth.

Notes: (A) Growth curve of parental cells and 23 I/XCRI cells in proliferation assay. Values shown are means \pm standard deviation (SD). * $p<0.05$, ** $p<0.0$ I. Data were from three independent experiments with triplicate samples. (B) We used T25 bottle for Anchorage independent colony formation on soft agar in association with XCRI protein expression, and the photos were taken by common camer, the number were showed in (C), Error bars $=95 \%$ Cls. Data from three independent experiments are shown. $* * p<0.01$. (D) Tumor growth curve of $23 \mathrm{I} /$ vector and $23 \mathrm{I} / \mathrm{XCR} \mathrm{I}$ cells in vivo. $* p<0.05, * * p<0.0 \mathrm{I}$. (E) Gross volume of $X C R \mathrm{I}$ over-expression tumors and vector control tumors. (F) The average rate of tumorigenesis in control xenografts and $23 \mathrm{I} / \mathrm{XCR}$ Ixenografts. $* * 0.0 \mathrm{I}$.

Abbreviation: OD, optical density.

P53 proteins, however levels of phosphorylated MEK, JNK, and $\mathrm{P} 38$ proteins were greatly decreased in 231/XCR1 versus controls, but phospo-P53 was increased in 231/XCR1 in vitro and in vivo (Figure 4A and 4B). We then tested the changes in $\mathrm{PI} 3 \mathrm{~K} / \mathrm{AKT} / \mathrm{mTOR}$ signaling pathways, the result showed in Figure $4 \mathrm{C}$ indicates that there was no change in total AKT protein level, but the activity of AKT phosphorylation was lower in 231/XCR1 than that of the parent cells. The decreased expression of total protein mTOR may have contributed to the decreased phosphorylated protein of $\mathrm{mTOR}$ in 231/XCR1 relative to controls. The proportion of P-P70S6K and P-4EBP1 proteins, which acted as the downstream targets of mTOR, also exhibited significantly small levels than the controls. LC3, especially the conversion of LC3-I (the upper band) to LC3-II (the lower band), reflected the concurrence of autophagy. We observed the upregulated conversion of LC3 in 231/XCR1, which was mostly due to the decreased activation of mTOR.

To confirm the observation, we also measured the expression of phospo-P53 and LC3 in the tissues generated from animals injected with $231 / \mathrm{XCR} 1$ or $231 /$ vector by immunohistochemistry. The results are shown in Figure 4B and $\mathrm{D}$ as expected, the levels of phospo-P53 and LC3 proteins are notably increased. Our results show that the decreased levels of MAPK and PI3K/AKT/mTOR pathways are associated with inhibited tumor growth induced by XCR1.

\section{$X C R I$ is associated with migration, invasion abilities of breast cancer cells}

In order to test whether XCR1 affect breast cancer cell migration and invasion, we performed transwell chambers assay. The result showed that more $231 / \mathrm{XCR} 1$ cells intruded into bottom chamber than 231/vector cells (Figure 5A-D). Thus, overexpression of XCR1 can increase cell migration and invasion in vitro.

We also investigated the metastasis of tumors using an orthotropic xenograft tumor model in BALB/c mice. The H\&E-stained sections were then subjected to microscopic examination for morphologic evidence of tumor cells by light microscopy. But no pulmonary metastasis or lymph node metastasis was observed on both $231 / \mathrm{XCR} 1$ and the control 
A

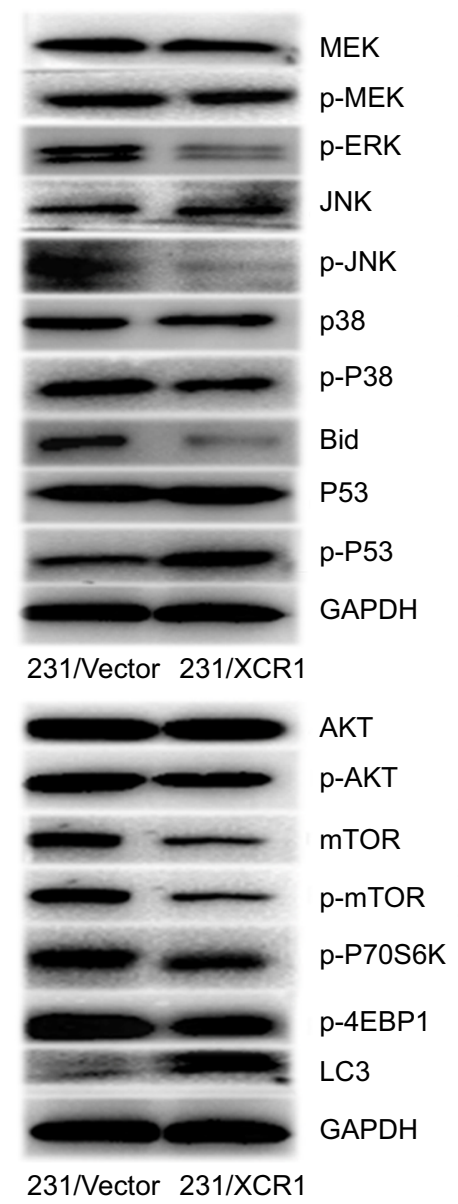

B

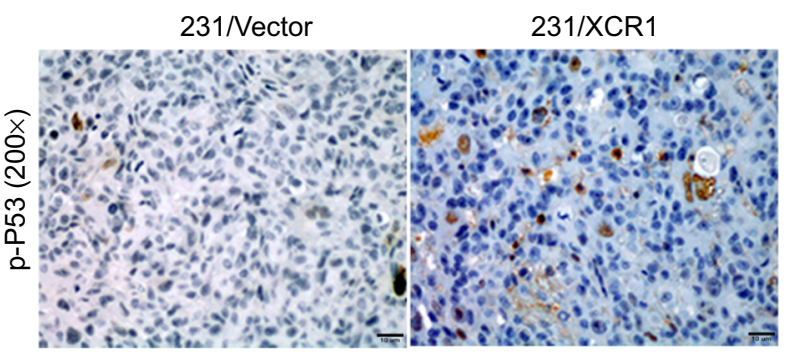

D

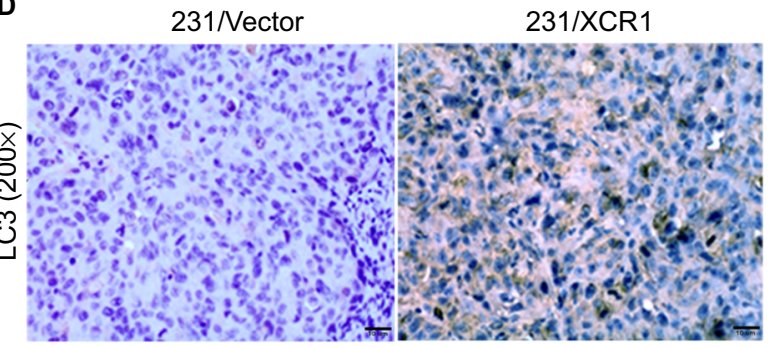

Figure 4 Activation of MAPK and PI3K/AKT/mTOR pathways is involved in XCRI-mediated tumor cell.

Notes: (A, C) Western blot analysis of MAPK and PI3K/AKT/mTOR pathways protein expression in 23I/vector and $23 \mathrm{I} / \mathrm{XCR}$ I cells. (B, D) Phospo-P53 and LC3 protein expression were detected by immunohistochemistry analysis in xenografts of $23 \mathrm{I} /$ vector and $23 \mathrm{I} / \mathrm{XCR} \mathrm{I}$ tumor bearing mice.

Abbreviations: GAPDH, glyceraldehyde 3-phosphate dehydrogenase; MAPK, mitogen-activated protein kinase; MEK, MAPK/ERK kinase.

cells. We further found that the level of protein $\beta$-catenin was downregulated in 231/XCR1 compared with 231 cells, this may be associated with the higher migration and invasion, which was mediated by XCR1. Collectively, these data suggest that $\mathrm{XCR} 1$ promotes breast cancer invasion and migration partly through decreasing the protein level of $\beta$-catenin (Figure 5E).

\section{Discussion}

Chemokines are a superfamily of small chemotactic cytokines, which have been classified into four main subfamilies on the basis of the relative position of the conserved two $\mathrm{N}$-terminal cysteine residues: $\mathrm{CXC}, \mathrm{CC}, \mathrm{CX} 3 \mathrm{C}$, and $\mathrm{XC}$ and control leukocyte migration and trafficking together with their receptors. ${ }^{17,18}$ It is now appreciated that chemokines and their receptors have a critical role in cancer cell growth and metastasis. For example, tumor hypoxia promotes the recruitment of regulatory $\mathrm{T}$ (Treg) cells through the induction of expression of CCL28, which, in turn, promotes tumor tolerance and angiogenesis. ${ }^{19}$ Chemokine receptor CXCR4 gene silencing can inhibit breast cancer metastasis to the lung in nude mice, ${ }^{20}$ and posttranscriptional regulation of CXCR4 by estrogens determines the metastatic potential of breast cancer cells. ${ }^{21}$

$\mathrm{XCR} 1$ is the only receptor for XCL1, and it is a typical seven transmembrane G-protein-coupled receptor, ${ }^{22} \mathrm{XCR} 1$ is expressed by a DC subpopulation. The interaction between XCL1 and XCR1 plays an important role in DC-mediated immune response and thymic development of Treg cells. ${ }^{2}$ There are more evidence about the important role of XCL1 in many tumors due to the significant immune response. For example, it reported that the strategy combined interleukin (IL)-10 with Lptn gene can improve the antitumor efficacy in EG7 tumor-bearing mice. ${ }^{1}$ Another study showed that autologous tumor cell vaccines combining transgenic lymphotactin with IL-2 seem to have little toxicity in humans and can induce an antitumor immune response. ${ }^{23}$ However, the function of the receptor in epithelial cancer has not been well defined.

Our data here showed a link between XCR1 and ER expression in breast cancer, that XCR1 expression level was 
A

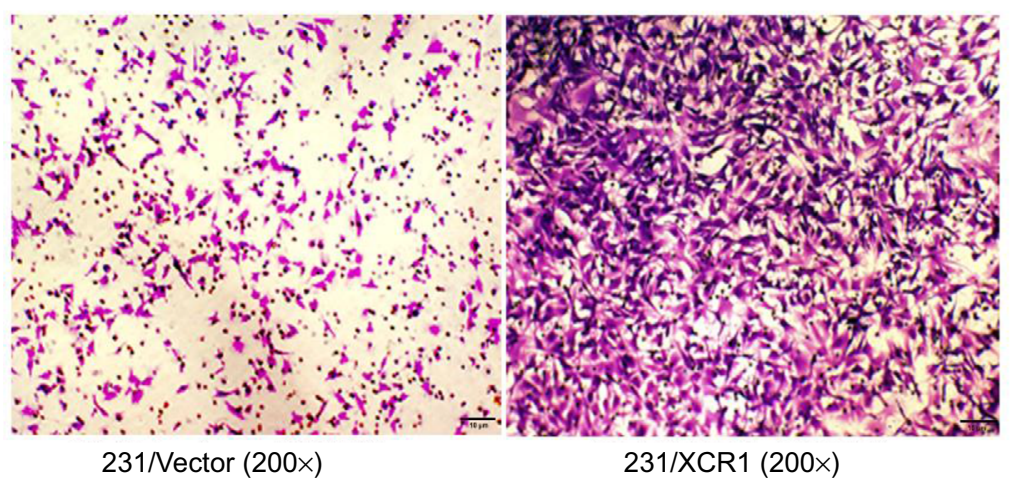

C

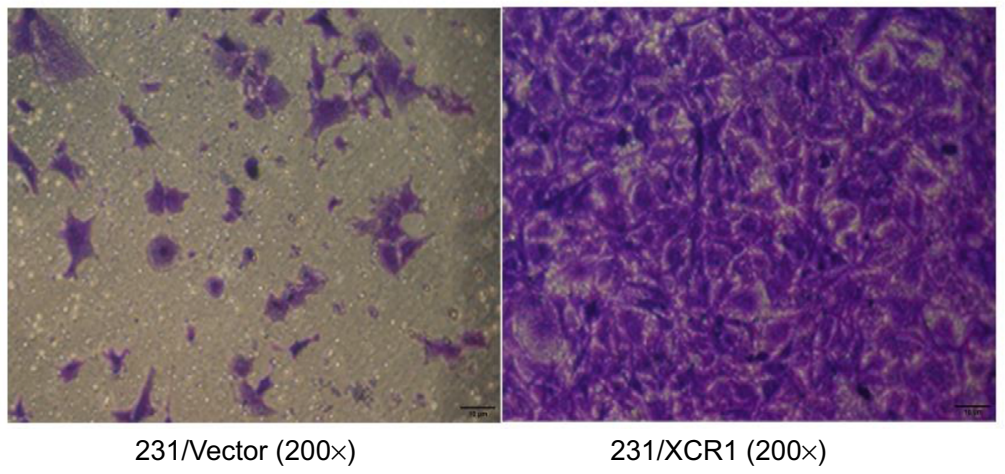

B
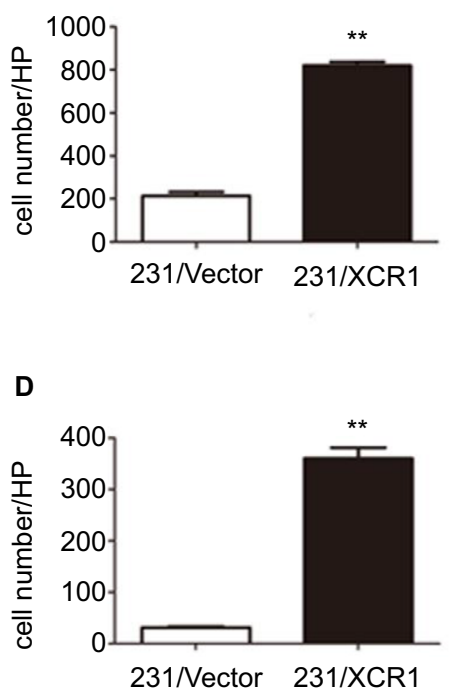

E

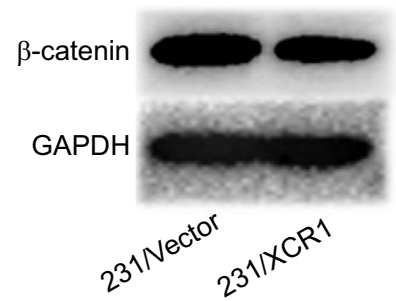

Figure 5 XCRI is associated with migration, invasion abilities of breast cancer cells.

Notes: Migration assay (A) and invasion assay (C) for $23 \mathrm{I} /$ vector and $23 \mathrm{I} / \mathrm{XCR}$ I cells plated on the upper cell culture inserts, with culture medium alone plated in the lower chambers. Quantification for the transwell assay (B, D). Cells were counted in triplicate well and in three identical experiments. $* *<<0.0$ I Columns mean of three independent experiments, bars standard deviation, HP (high-power objective). (E) The level of protein $\beta$-catenin was examined by Western blot.

Abbreviation: GAPDH, glyceraldehyde 3-phosphate dehydrogenase.

significantly higher in ER+ breast cancer cell lines (MCF-7, T47D) than ER- cell lines (MDA-MB-231, MDA-MB-468). We further confirmed the strong association by immunohistochemistry in 10 formalin-fixed, paraffin-embedded breast cancer tissue sections. However, our results are based on a limited number of cases, and further work of large samples is warranted. Keen et al reported that XCL1 appears to be ER responsive and downstream of ER expression by utilizing newly developed genome-wide screening microarray techniques. ${ }^{24}$ Different results may be due to the different roles of XCL1 and XCR1 on ER, but they all indicate important association with ER.

We then used a model of XCR1 overexpression MDAMB-231 breast cancer to identify the functional role of $\mathrm{XCR} 1$ in breast cancer. We found that overexpression of XCR1 in MDA-MB-231 cell line inhibited cell proliferation, tumorigenesis, and tumor growth, but promoted invasiveness and tumor metastasis. This is different with the previous study in oral squamous cell carcinoma and human EOC. They showed that XCR1 is expressed early during the course of tumorigenic transformation and contributes toward increased cell migration and proliferation, which can facilitate the prometastatic behavior of EOC cells. ${ }^{13}$ XCL1 mediated intracellular activation of the ERK1/2 signaling pathway and stimulated migration, invasion, and proliferation of all cells through XCR $1 .{ }^{12}$ Different results may be due to the different source of XCR1 and the close association of ER, which deserve to be further investigated in the future.

The role of MAPK and PI3K/AKT/mTOR signaling pathways in the proliferation of breast cancer has previously been reported, ${ }^{25-29}$ so we studied the activation of these two signaling pathways. We found that the levels of phosphorylated MEK, JNK, P38, protein were greatly decreased but P53 increased in 231/XCR1 versus controls, which raise the possibility that XCR1 may suppress tumor growth by reducing activation of MAPK signaling pathways. The mTOR pathway plays an important role in supporting cell growth, survival, and endocrine resistance. ${ }^{30,31}$ ER stress negatively regulates $\mathrm{AKT} / \mathrm{TSC} / \mathrm{mTOR}$ pathway to enhance autophagy. ${ }^{32}$ The downstream effectors of mTOR pathway, such as p70S6K, 4E-BP1 that control 5'TOP mRNA and cap-dependent mRNA translation of HIF1a in autophagy. 
The results report that the decreased levels of phosphorylated mTOR were found in XCR1-overexpressing breast cancer cell. Our data indicate that reduced cell growth also correlates with decreased activation of the MTOR pathway in 231/XCR1 tumors, which is based on results showing significantly smaller levels of P-p70-S6K in 231/XCR1 tumors relative to controls in vitro. Furthermore, we documented the mechanism that XCR1 promotes cell invasiveness and tumor metastasis partly due to suppressing the expression of cell adhesion protein $\beta$-catenin.

Two-thirds of breast cancer overexpress ER and/or progesterone receptors, which typically suggest a degree of estrogen dependence for growth. ${ }^{30}$ It has been reported that the development of estrogen independence and endocrine therapy resistance in $\mathrm{BC}$ patients may be correlated with SDF-1-CXCR4 signaling, ${ }^{33-35}$ the insulin-like growth factor (IGF) system, ${ }^{36}$ IGF1 receptor (IGF1R) expression, ${ }^{37}$ and hyperactivation of the IGF-IR/InsR/PI3K/mTOR pathway. ${ }^{38}$ Here, we found a strong association between XCR1 and ER, the further biological effects of XCR 1 in breast cancer are that XCR1 can inhibit cell growth and tumorigenesis via downregulating the activation of MAPK and PI3K/AKT/mTOR signaling pathway but promote the migration and invasion by decreasing the protein level of $\beta$-catenin. It seems completely contradictory to each other, but in clinic, sometimes we first check out the metastatic tumor without finding the prime cancer, we guess there must be some genes, such as XCR1, that just affect on tumor migration and invasion but decrease the tumor growth. We believe controlling the expression of XCR1 in different steps of tumor development that can effectively inhibit breast cancer, especially ER+ cell growth and metastasis that may be a hopeful therapeutic strategy against estrogen independence and tumor relapse in future, but further studies of XCR1 are still needed.

\section{Acknowledgment}

This study was supported by the National Natural Science Foundation of China (Grant No. 81172506) and the Shanghai Committee of Science and Technology, People's Republic of China (Grant No. 12DZ2260100).

\section{Disclosure}

The authors report no conflicts of interest in this work.

\section{References}

1. Zhang J, Zhou Z, Wang C, et al. Reduced tumorigenesis of EG7 after interleukin-10 gene transfer and enhanced efficacy in combination with intratumorally injection of adenovirus-mediated lymphotactin and the underlying mechanism. Cancer Immunol Immunother. 2011;60(4):559-573.
2. Lei Y, Takahama Y. XCL1 and XCR1 in the immune system. Microbes Infect. 2012;14(3):262-267.

3. Kumar S, Choi WT, Dong CZ, et al. SMM-chemokines: a class of unnatural synthetic molecules as chemical probes of chemokine receptor biology and leads for therapeutic development. Chem Biol. 2006; 13(1):69-79.

4. Dong C, Chua A, Ganguly B, Krensky AM, Clayberger C. Glycosylated recombinant human XCL1/lymphotactin exhibits enhanced biologic activity. J Immunol Methods. 2005;302(1-2):136-144.

5. Bachem A, Hartung E, Güttler S. Expression of XCR1 characterizes the Batf3-dependent lineage of dendritic cells capable of antigen crosspresentation. Front Immunol. 2012;3:214.

6. Yamazaki C, Miyamoto R, Hoshino K, et al. Conservation of a chemokine system, XCR1 and its ligand, XCL1, between human and mice. Biochem Biophys Res Commun. 2010;397(4):756-761.

7. Nguyen KD, Fohner A, Booker JD, Dong C, Krensky AM, Nadeau KC. XCL1 enhances regulatory activities of CD4+ CD25(high) CD127(low/-) T cells in human allergic asthma. J Immunol. 2008;181(8): 5386-5395.

8. Yeh PT, Lin FA, Lin CP, Yang CM, Chen MS, Yang CH. Expressions of lymphotactin and its receptor, XCR, in Lewis rats with experimental autoimmune anterior uveitis. Graefes Arch Clin Exp Ophthalmol. 2010;248(12):1737-1747.

9. Endres M, Andreas K, Kalwitz G, et al. Chemokine profile of synovial fluid from normal, osteoarthritis and rheumatoid arthritis patients: CCL25, CXCL10 and XCL1 recruit human subchondral mesenchymal progenitor cells. Osteoarthritis Cartilage. 2010;18(11):1458-1466.

10. Ordway D, Higgins DM, Sanchez-Campillo J, et al. XCL1 (lymphotactin) chemokine produced by activated $\mathrm{CD} 8 \mathrm{~T}$ cells during the chronic stage of infection with Mycobacterium tuberculosis negatively affects production of IFN-gamma by CD4 T cells and participates in granuloma stability. J Leukoc Biol. 2007;82(5):1221-1229.

11. Seow SW, Rahmat JN, Bay BH, Lee YK, Mahendran R. Expression of chemokine/cytokine genes and immune cell recruitment following the instillation of mycobacterium bovis, bacillus calmette-guerin or lactobacillus rhamnosus strain GG in the healthy murine bladder. Immunology. 2008;124(3):419-427.

12. Khurram SA, Whawell SA, Bingle L, Murdoch C, McCabe BM, Farthing PM. Functional expression of the chemokine receptor XCR1 on oral epithelial cells. J Pathol. 2010;221(2):153-163.

13. Kim M, Rooper L, Xie J, et al. The lymphotactin receptor is expressed in epithelial ovarian carcinoma and contributes to cell migration and proliferation. Mol Cancer Res. 2012;10(11):1419-1429.

14. Yamashita Y, Kajiura D, Tang L, et al. XCR1 expression and biased VH gene usage are distinct features of diffuse large B-cell lymphoma initially manifesting in the bone marrow. Am J Clin Pathol. 2011:135(4):556-564.

15. Li JY, Ou ZL, Yu SJ, et al. The chemokine receptor CCR4 promotes tumor growth and lung metastasis in breast cancer. Breast Cancer Res Treat. 2012;131(3):837-848.

16. Jones LW, Viglianti BL, Tashjian JA, et al. Effect of aerobic exercise on tumor physiology in an animal model of human breast cancer. $J$ Appl Physiol. 2010;108(2):343-348.

17. Rossi D, Zlotnik A. The biology of chemokines and their receptors. Annu Rev Immunol. 2000;18:217-242.

18. Caux C, Ait-Yahia S, Chemin K, et al. Dendritic cell biology and regulation of dendritic cell trafficking by chemokines. Springer Semin Immunopathol. 2000;22(4):345-369.

19. Facciabene A, Peng X, Hagemann IS, et al. Tumour hypoxia promotes tolerance and angiogenesis via CCL28 and T (reg) cells. Nature. 2011;475(7355):226-230.

20. Zhang MX, Han N, Yu SY, Leng Y. [Chemokine receptor CXCR4 gene silencing with shRNA inhibits breast cancer metastasis to the lung in nude mice]. Zhonghua Zhong Liu Za Zhi. 2008;30(5):325-329. Chinese.

21. Sengupta S, Schiff R, Katzenellenbogen BS. Post-transcriptional regulation of chemokine receptor CXCR4 by estrogen in HER2 overexpressing, estrogen receptor-positive breast cancer cells. Breast Cancer Res Treat. 2009;117(2):243-251. 
22. Yoshida, T, Imai T, Kakizaki M, Nishimura M, Takagi S, Yoshie O. Identification of single $\mathrm{C}$ motif-1/lymphotactin receptor XCR1. J Biol Chem. 1998;273(26):16551-16554.

23. Russell HV, Strother D, Mei Z, et al. Phase I trial of vaccination with autologous neuroblastoma tumor cells genetically modified to secrete IL-2 and lymphotactin. J Immunother. 2007;30(2):227-233.

24. Keen JC, Garrett-Mayer E, Pettit C, et al. Epigenetic regulation of protein phosphatase 2A (PP2A), lymphotactin (XCL1) and estrogen receptor alpha (ER) expression in human breast cancer cells. Cancer Biol Ther. 2004;3(12):1304-1312.

25. Sos ML, Fischer S, Ullrich R, et al. Identifying genotype-dependent efficacy of single and combined PI3K- and MAPK-pathway inhibition in cancer. Proc Natl Acad Sci U S A. 2009;106(43):18351-18356.

26. Mirzoeva OK, Das D, Heiser LM, et al. Basal subtype and MAPK/ERK kinase (MEK)-phosphoinositide 3-kinase feedback signaling determine susceptibility of breast cancer cells to MEK inhibition. Cancer Res. 2009;69(2):565-572.

27. Qi X, Zhi H, Lepp A, et al. p38gamma mitogen-activated protein kinase (MAPK) confers breast cancer hormone sensitivity by switching estrogen receptor (ER) signaling from classical to nonclassical pathway via stimulating ER phosphorylation and c-Jun transcription. J Biol Chem. 2012;287(18):14681-14691.

28. Chen J, Sun L. Formononetin-induced apoptosis by activation of Ras/ p38 mitogen-activated protein kinase in estrogen receptor-positive human breast cancer cells. Horm Metab Res. 2012;44(13):943-948.

29. Liu X, Wang L, Chen J, et al. Estrogen receptor beta agonist enhances temozolomide sensitivity of glioma cells by inhibiting PI3K/AKT/ mTOR pathway. Mol Med Rep. 2015;11(2):1516-1522.
30. De P, Miskimins K, Dey N, Leyland-Jones B. Promise of rapalogues versus mTOR kinase inhibitors in subset specific breast cancer: old targets new hope. Cancer Treat Rev. 2013;39(5):403-412.

31. Seto B. Rapamycin and mTOR: a serendipitous discovery and implications for breast cancer. Clin Transl Med. 2012;1(1):29.

32. Qin L, Wang Z, Tao L, Wang Y. ER stress negatively regulates AKT/TSC/ mTOR pathway to enhance autophagy. Autophagy. 2010;6(2):239-247.

33. Rhodes LV, Short SP, Neel NF, et al. Cytokine receptor CXCR4 mediates estrogen-independent tumorigenesis, metastasis, and resistance to endocrine therapy in human breast cancer. Cancer Res. 2011;71(2):603-613.

34. Liang Z, Brooks J, Willard M, et al. CXCR4/CXCL12 axis promotes VEGF-mediated tumor angiogenesis through Akt signaling pathway. Biochem Biophys Res Commun. 2007;359(3):716-722.

35. Dragowska WH, Verreault M, Yapp DT, et al. Decreased levels of hypoxic cells in gefitinib treated ER+ HER-2 overexpressing MCF-7 breast cancer tumors are associated with hyperactivation of the mTOR pathway: therapeutic implications for combination therapy with rapamycin. Breast Cancer Res Treat. 2007;106(3):319-331.

36. Casa AJ, Dearth RK, Litzenburger BC, Lee AV, Cui X. The type I insulinlike growth factor receptor pathway: a key player in cancer therapeutic resistance. Front Biosci. 2008;13:3273-3287.

37. Maor S, Mayer D, Yarden RI, et al. Estrogen receptor regulates insulin-like growth factor-I receptor gene expression in breast tumor cells: involvement of transcription factor Sp1. J Endocrinol. 2006;191(3):605-612.

38. Miller TW, Hennessy BT, González-Angulo AM, et al. Hyperactivation of phosphatidylinositol-3 kinase promotes escape from hormone dependence in estrogen receptor-positive human breast cancer. J Clin Invest. 2010;120(7):2406-2413.
Breast Cancer - Targets and Therapy

\section{Publish your work in this journal}

Breast Cancer - Targets and Therapy is an international, peerreviewed open access journal focusing on breast cancer research, identification of therapeutic targets and the optimal use of preventative and integrated treatment interventions to achieve improved outcomes, enhanced survival and quality of life for the cancer patient.

\section{Dovepress}

The manuscript management system is completely online and includes a very quick and fair peer-review system, which is all easy to use. Visit http://www.dovepress.com/testimonials.php to read real quotes from published authors. 